\section{Efficacy of a combination of sodium valproate and baclofen in Meige's disease (idiopathic orofacial dystonia)}

Meige's disease is a bizarre, often disabling movement disorder of the elderly characterised by symmetric dystonic contractions of the orofacial muscles; at times other midline muscles such as the tongue and the palatopharyngeal, respiratory, and neck muscles may be affected. ${ }^{12}$ Although the spasms are often thought to be psychogenic in origin, there exists no convincing evidence that they represent a manifestation of a psychiatric disorder. ${ }^{1}$ Similarly, there is little information on the pathophysiology of the disorder. Neophytides et $a l^{3}$ reported decreased cerebrospinal fluid $\gamma$-aminobutyrate concentrations in patients with adult-onset dystonia. If this reflects a reduction in central $\gamma$-aminobutyric-acid-mediated inhibition, the syndrome might result from a striatal dopaminergic predominance secondary to disinhibition of dopaminergic nigral neurons. Indeed, drugs with dopamine-receptor-blocking properties have been used, with limited success, for the dystonic spasms. ${ }^{4}$ We report a good clinical response in one case of Meige's disease treated with the $\gamma$ aminobutyric-acid-mimetic combination of sodium valproate and baclofen.

\section{Case report}

A 56-year-old black man presented with a three-year history of a prom inent facial dystonia that was not part of a well-known neurological disorder. He had been treated previously with haloperidol, pimozide, and diazepam; neither haloperidol nor pimozide had produced noticeable improvement; diazepam had alleviated the involuntary spasms considerably but produced drowsiness to the extent that he spent most of the day sleeping. At the time of evaluation he had received no medication for three months.

A detailed medical and family history was obtained from the patient, his sister, and his hospital records. Specific inquiries about siblings, children parents, aunts and uncles, and grandparents did not disclose any familial history of movement disorders. Examination of the sister showed nothing abnormal. There was no history of administration of neuroleptics before the onset of the disorder. Results of general systemic examination were within normal limits. The types of spasm observed in the patient were mild bilateral blepharospasm, mouth retraction, eyebrow raising and frowning, jaw opening, mouth clenching, lip pursing and tightening, platysmal contractions and neck spasms (lateral rotation), and spasms of flexion of the right fingers. He had dysarthria but no appreciable dysphagia. The dystonic movements were aggravated by stress or emotion and often initiated by speaking. Spasms were not observed during sleep. The patient also often complained of deep pain in the region of the left ear; indeed, this symptom was subjectively the most distressing. He had no other neurological abnormality, and mental state examination showed no evidence of dementia or psychiatric disorder. No specific cause for the dystonia was found despite an extensive work-up, which included routine serum biochemical estimations; thyroid function studies; measurement of serum caeruloplasim, vitamin $B_{12}$, and folate concentrations; skull radiography; electroencephalography; computed tomography; and examination of cerebrospinal fluid. Based on these findings Meige's syndrome was diagnosed. ${ }^{12}$

Treatment with a combination of sodium valproate (Epilim) and baclofen (Lioresal) was started and the doses increased over -10 days to $2400 \mathrm{mg}$ and $90 \mathrm{mg}$ daily respectively in three divided doses. After 14 days the frequency and severity of the dystonic spasms were greatly reduced, though occasional spasms affecting the buccal and platysmal muscles were still present. The patient reported a pronounced subjective improvement and no longer complained of ear pain. Eight weeks later the condition remained in remission and no side effects or toxic effects of treatment were noted.

\section{Comment}

Though its precise mechanism of action is not known, sodium valproate is thought to act by increasing the synaptic concentration of $\gamma$-aminobutyric acid in the brain; baclofen has recently been shown to be a selective agonist for $\gamma$-aminobutyric acid B receptors which are present on central nerve terminals and through which the release of several transmitters, including dopamine, appears to be inhibited. ${ }^{5}$ Thus a combination of the two drugs could be expected to act synergistically, reducing activity in the nigrostriatal dopaminergic pathway and inhibiting release of dopamine from dopaminergic terminals in the striatum. Such a combination of actions might represent a more physiological means of reducing striatal dopaminergic preponderance than simple blockade of dopamine receptors. Although this hypothesis must be tested, our report of substantial improvement in a case of Meige's disease treated with sodium valproate and baclofen suggests that a more extensive and prolonged trial of these agents is warranted in this distressing and often intractable disorder.

Requests for reprints should be sent to MJWB.

Marsden CD. Blepharospasm-oromandibular dystonia syndrome (Brueghel's syndrome). 7 Neurol Neurosurg Psychiatry 1976;39:1204-9.

2 Tolosa ES. Clinical features of Meige's disease (idiopathic orofacial dystonia). Arch Neurol 1981;38:147-51.

${ }^{3}$ Neophytides A, Suria A, Chase TN. Cerebrospinal fluid GABA in neurological disease. Neurology 1978;28:359.

4 Tolosa ES, Lai C. Meige's disease: striatal dopaminergic predominance. Neurology $1979 ; 29: 1126-30$

${ }^{5}$ Hill DR, Bowery NG. ${ }^{3} \mathrm{H}$-baclofen and ${ }^{3} \mathrm{H}-\mathrm{GABA}$ bind to bicucullineinsensitive $\mathrm{GABA}_{\mathrm{B}}$ sites in rat brain. Nature 1981;290:149-52.

(Accepted 11 fune 1982)

MRC Brain Metabolism Research Laboratory, University of the Witwatersrand and the South African Institute for Medical Research, Johannesburg 2001, South Africa

$M \mathrm{~J}$ W BRENNAN, $\mathrm{MB}$, PHD, principal investigator (present address: Neurological Unit, Boston City Hospital, Boston, Mass 02118 USA)

$P$ RUFF, $M B, B C H$, registrar

R SANDYK, MD, registrar

\section{Rising female predominance in incidence of gastric ulcer}

Over the past five decades there has been a remarkable change in the pattern of frequency of gastric and duodenal ulcers. For example, it has been suggested that the overall incidence of peptic ulcers has been steadily decreasing. Many analyses have used "peptic ulcer" as one disease and not recognised gastric and duodenal ulcers as separate entities. Most of the available data have been based on morbidityfor example, perforated peptic ulcer-or mortality figures ${ }^{1}$ and tend to give a biased sampling when compared with a survey using radiographic or endoscopic techniques, or both.

\section{Patients, methods, and results}

We analysed retrospectively all the patients who had undergone upper gastrointestinal endoscopy in the department of gastroenterology at Auckland Hospital from January 1974 to December 1981. Patients (outpatients and inpatients) with gastric ulcers were selected and classified into type I (ulcers in the body), type II (gastric and duodenal ulcers in combination), and type III (ulcers in the antrum). Ulcers in the pyloric channel, malignant gastric ulcers, and gastric ulcers in association with previous gastric surgery were excluded. The table gives the distribution of patients with benign gastric ulcers. The overall male to female ratio was $0 \cdot 7: 1$. When further divided into subgroups, the overall female predominance could be exclusively accounted for by the striking male to female ratio of $0 \cdot 2: 1$ in the group with type I gastric ulcer. Patients with type I gastric ulcers also tended to be older, but the difference was not statistically significant ( $p>0.4 ; \chi^{2}$ test).

There were 787 patients with duodenal ulcers (male to female ratio $3 \cdot 7: 1$, mean age 47.2 SD 9.3 years). The duodenal to gastric ulcer ratio was $3 \cdot 1: 1$. Forty-two patients had histologically or cytologically confirmed carcinomas, and 10 of these were associated with an ulcer.

Clinical data and grouping of patients with benign gastric ulcers

\begin{tabular}{lccc}
\hline & \multicolumn{3}{c}{ Gastric ulcers $(n=242)$} \\
\cline { 2 - 4 } & Type I & Type II & Type III \\
\hline No ("i) of patients & $82(34)$ & $37(15)$ & $123(51)$ \\
Mean age \pm SD (years) & $63 \pm 11 \cdot 0$ & $59 \pm 0 \cdot 7$ & $52 \pm 1 \cdot 2$ \\
Male to female ratio & $0 \cdot 2: 1$ & $1 \cdot 3: 1$ & $1 \cdot 1: 1$
\end{tabular}

\section{Comment}

These results show a dramatic reversal of the male predominance in gastric ulcers over the past several decades. It is extremely difficult to compare various epidemiological data on gastric ulcer, but a slow 